\title{
Reduced Models for Uncertainty Quantification in the Cardiovascular Network via Domain Decomposition
}

\author{
S. Guzzetti ${ }^{1}$, L. A. Mansilla Alvarez ${ }^{2}$, P. J. Blanco ${ }^{2}$, K. T. Carlberg ${ }^{3}$, and A. Veneziani ${ }^{1}$ \\ ${ }^{1}$ Emory College, Department of Mathematics and Computer Science, 400 Dowman Dr., Atlanta GA 30322 \\ ${ }^{2}$ Laboratório Nacional de Computação Científica, Getúlio Vargas Av., 333, Quitandinha Petrópolis - Rio de Janeiro 25651-075, Brasil \\ ${ }^{3}$ Sandia National Laboratories, 7011 East Avenue, Livermore CA 94550
}

MOTIVATION
$\begin{aligned} & \text { Nacionalde } \\ & \text { Computação } \\ & \text { Científica }\end{aligned}$

\section{OBJECTIVES}

- Designing ad-hoc solvers for UQ in the cardiovascular system (CVS) that promote parallelism and scalability.

\section{UQ via DOMAIN DECOMPOSITION}

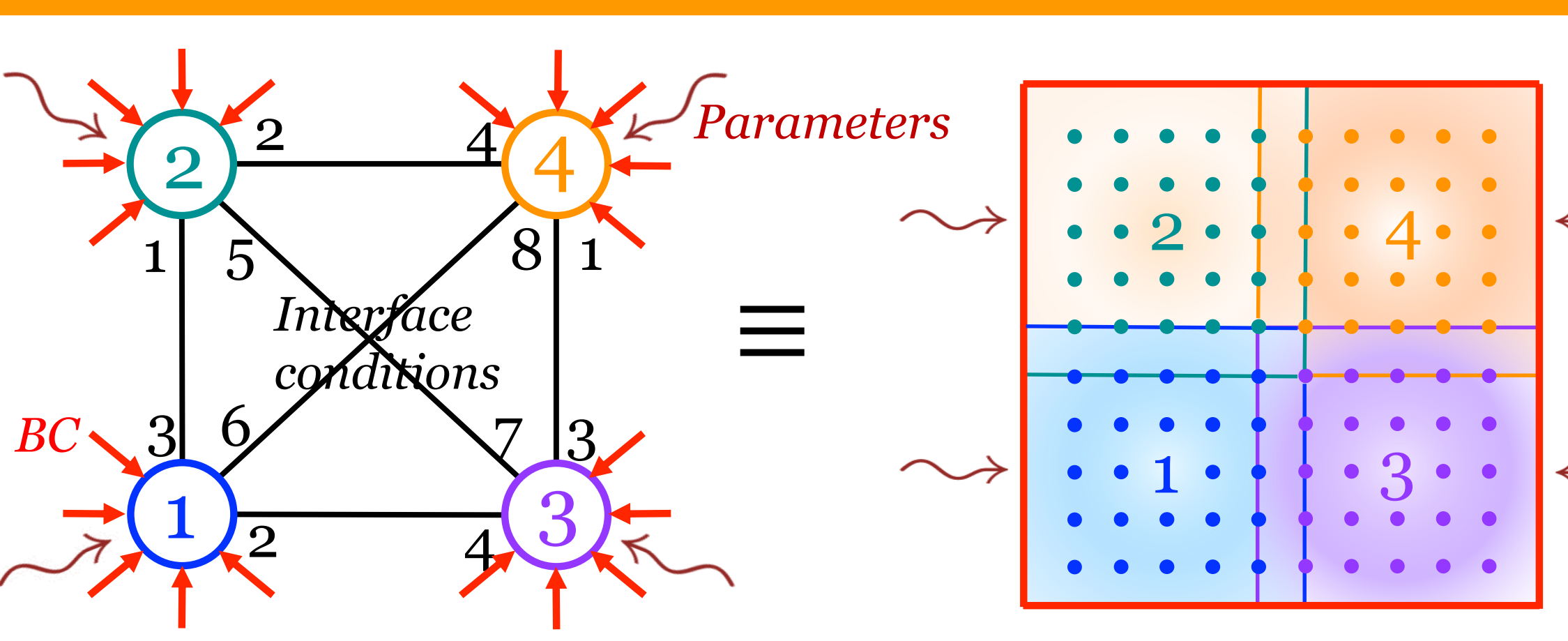

The Domain Decomposition Uncertainty Quantification (DDUQ) approach [1] performs UQ at the subsystem level, and propagates polynomial chaos coefficients via DD.

NUMERICAL RESULTS: 2D steady non-linear heat equation Accuracy: the error is bounded and independent of the distance from the boundary (left fig.).

Scalability: computational time is reduced for different relaxation schemes (right fig.).

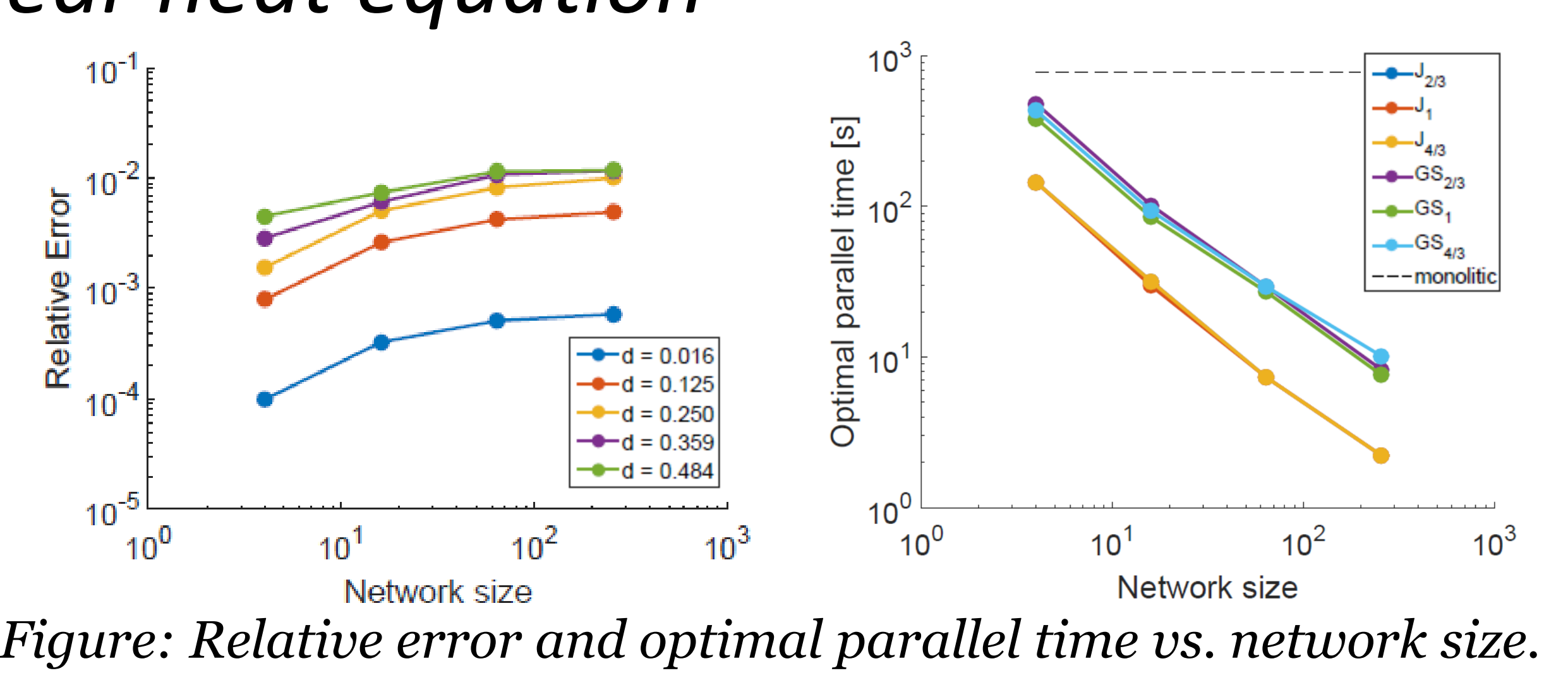

Enhancing the accuracy and reliability of the 1D reduced models, approximately at the same computational cost.

\section{"EDUCATED" REDUCED MODELS}

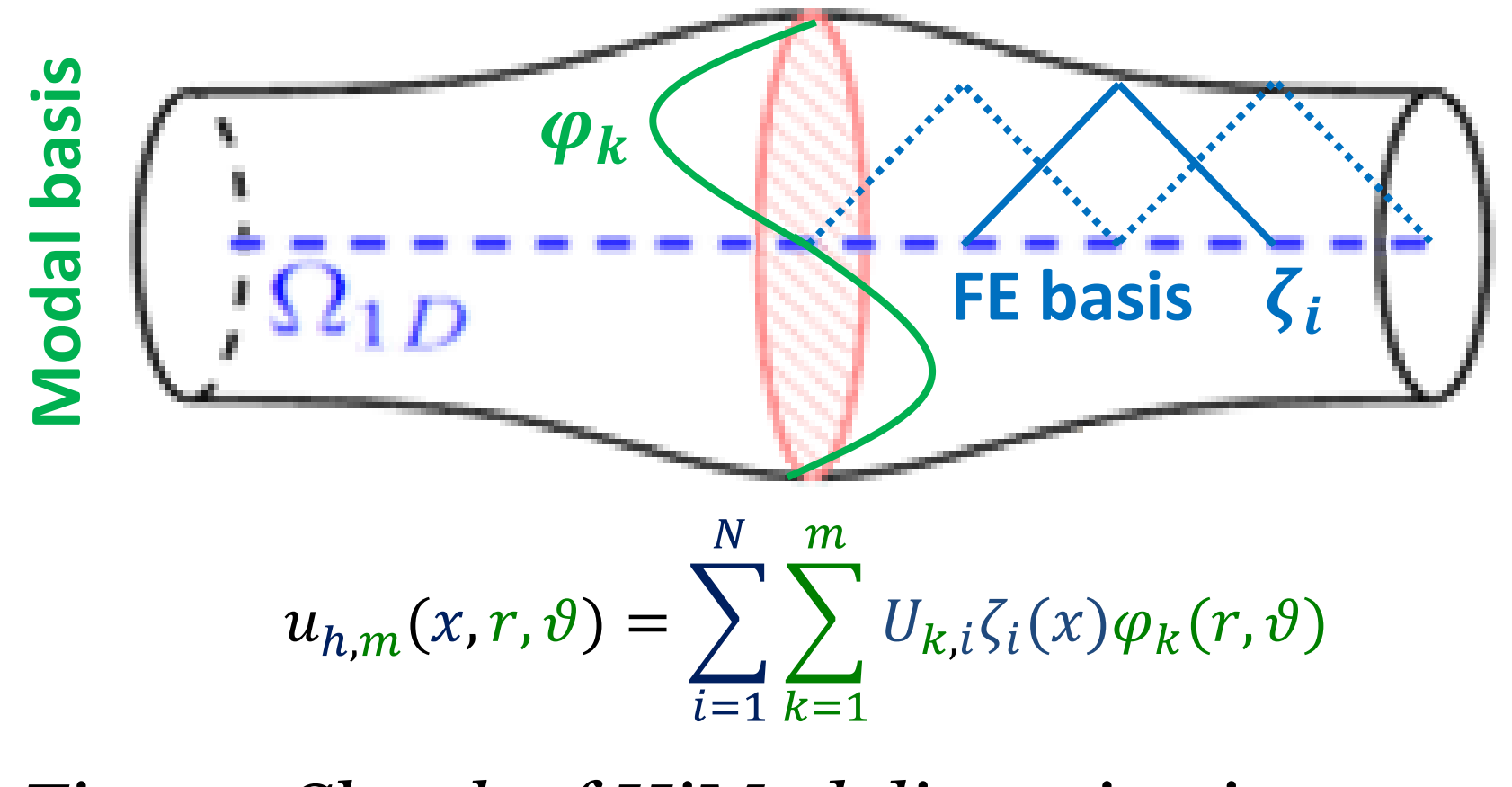

Figure: Sketch of HiMod discretizatio
The local cross-sectional dynamics discarded by 1D models can be retrieved via educated reduced models such as the Transversally Enriched Pipe Element Method (TEPEM) [2], or Hierarchical Model (HiMod) reduction methods [3].

\section{NUMERICAL RESULTS: 3D unsteady Navier-Stokes equations}

Accuracy of 3D solvers can be achieved at computational cost comparable to 1D models (left). Local transverse dynamics is preserved (right).

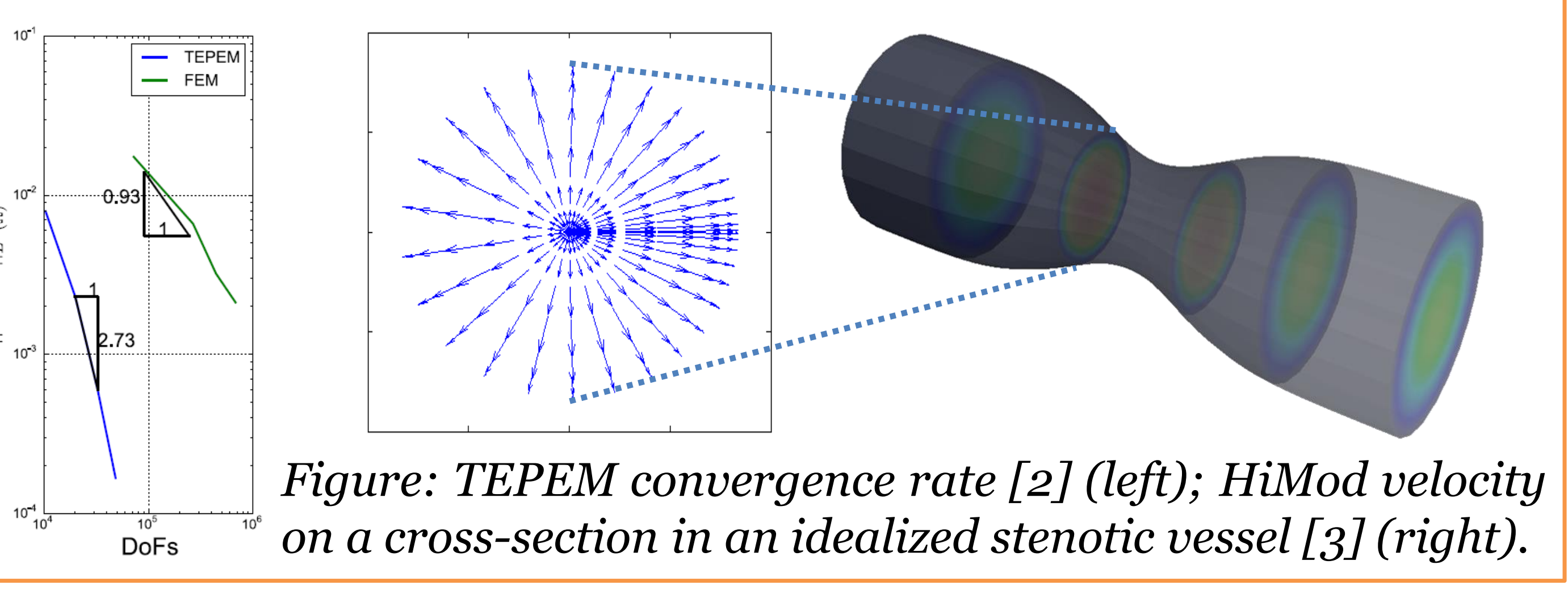

\section{UQ in the CARDIOVASCULAR NETWORK via DD}

\section{DETERMINISTIC 1D REDUCED MODEL}

\section{Bifurcation}

- Balance of fluxes $(Q)$

- Continuity of pressure $\left(P_{T}\right)$

- Extrapolation of Riemann invariants $\left(W_{1,2}\right)$

$\left\{\begin{array}{l}\sum_{i=1}^{N_{d}+1} Q_{i}=0 \\ P_{T, 1}=P_{T, i+1}, \quad i=1: N_{d} \\ W_{1,1}=W_{1,1}^{\star}, \quad W_{2, i}=W_{2, i}^{\star}\end{array}\right.$

Vessel (1D Euler equations)

Conservation law of mass and momentum averaged on the cross-section:

$\left\{\begin{array}{l}\frac{\partial A}{\partial t}+\frac{\partial Q}{\partial x}=0 \\ \frac{\partial Q}{\partial t}+\frac{\partial}{\partial x}\left(\frac{Q^{2}}{A}\right)+\frac{A}{\rho} \frac{\partial p}{\partial x}+K_{R} \frac{Q}{A}=0\end{array}\right.$

\section{DDUQ NUMERICAL MODEL}

for $n=1, \ldots, N_{t}$ do

Update exogenous inputs

for $n_{b}=1, \ldots, N_{b}$ do

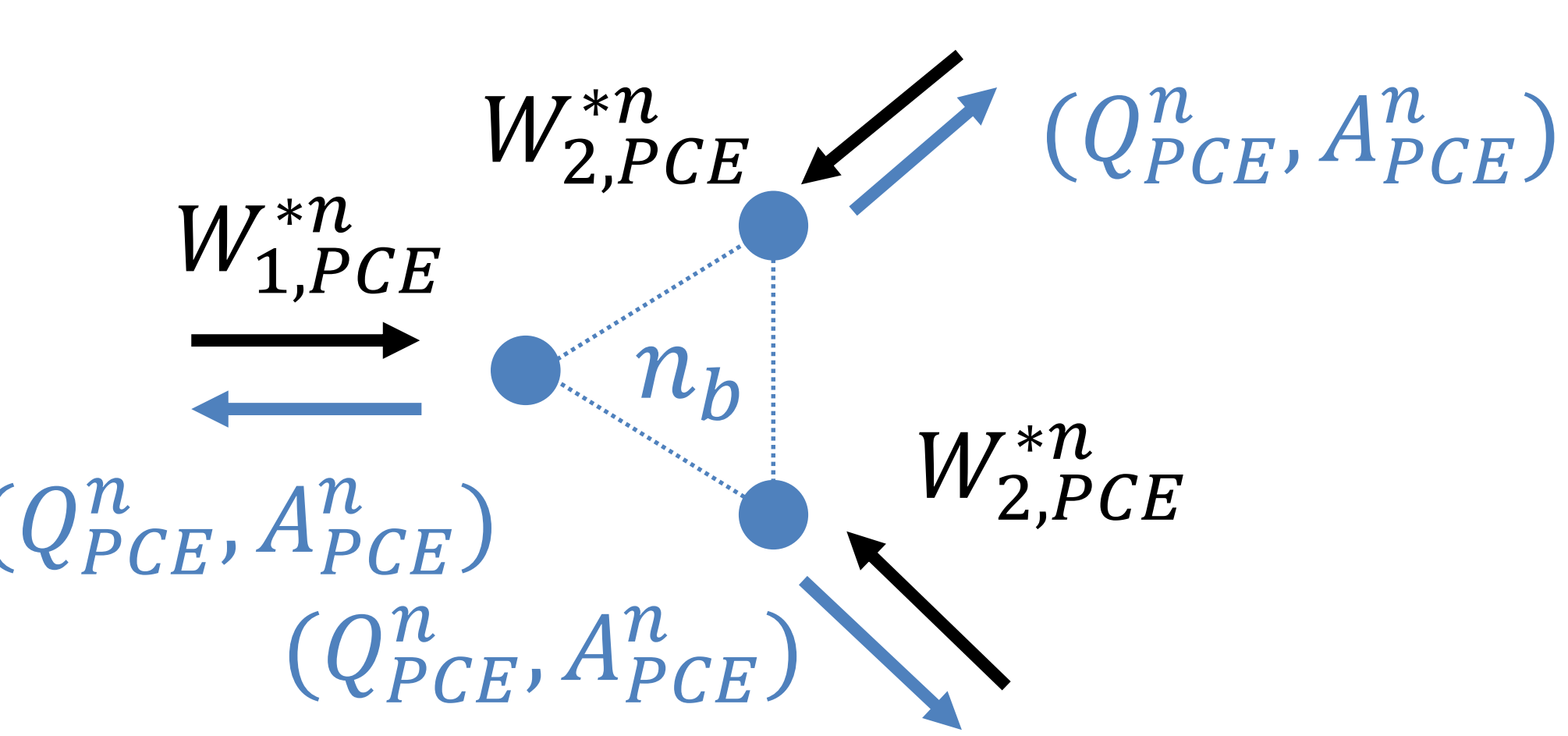

end for

for $n_{v}=1, \ldots, N_{v}$ do

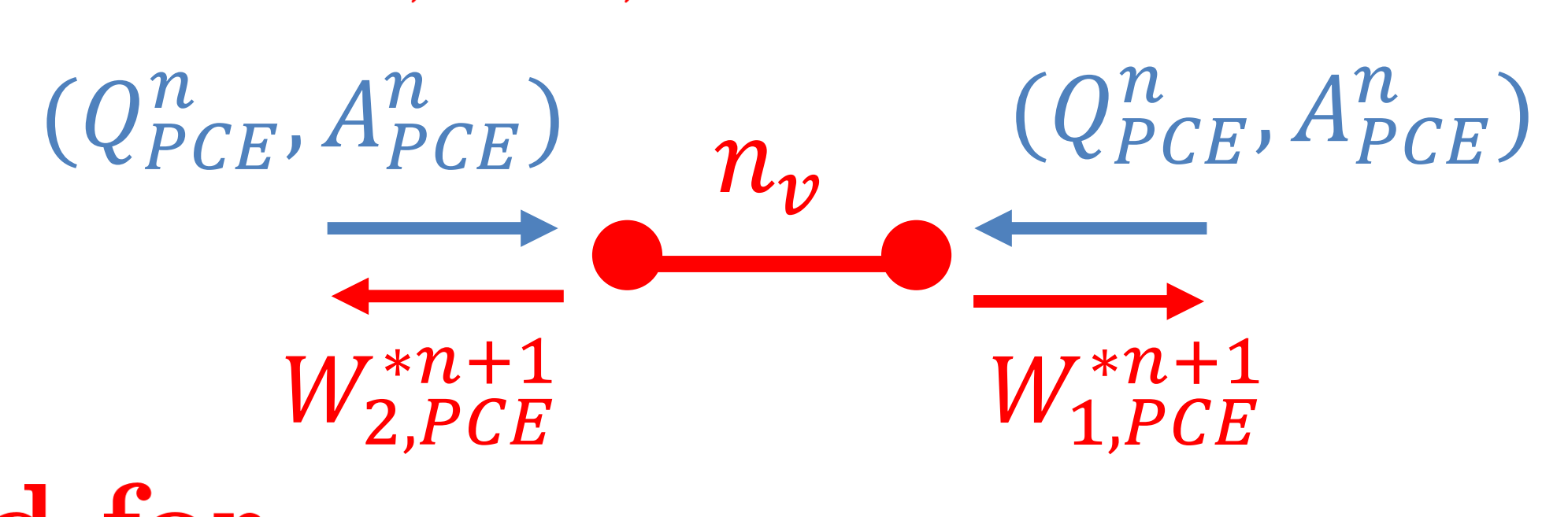

end for end for

\section{NUMERICAL VALIDATION}

Pulse wave travelling in a tapered bifurcated vessel with reflecting $B C$ and 3 stochastic parameters.

Good agreement with the literature [4].
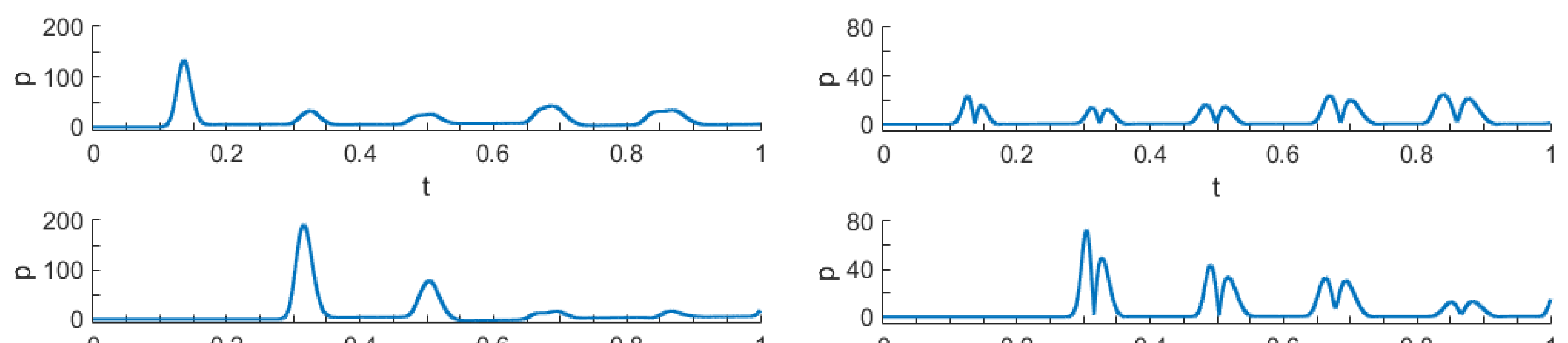

Figure: Mean (left) and STD (right) of pressure for parent (top) and daughter (bottom) vessel at the center of the pipe.
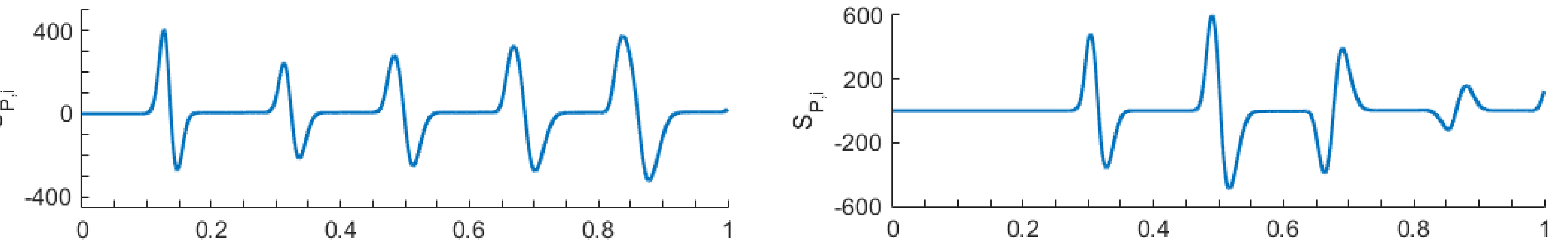

Figure: Sensitivity of pressure with respect to the stochastic parameter $\beta$ in the parent (left) and daughter (right) vessel at the center of the corresponding pipe.

\section{CONCLUSIONS and FUTURE WORK}

\section{DISCUSSION}

- The computational cost of UQ in the cardiovascular system can be reduced by promoting the independence of the subsystems and avoiding fullsystem simulations;

- The accuracy of 1D solvers can be improved by educated reduced models, at roughly the same computational cost.

\section{WORK IN PROGRESS}

- DDUQ numerical simulations in real cardiovascular networks;

- Embedding of the educated reduced models in the DDUQ solver;

- Parallelization and scalability test;

- Comparison with 1D models in terms of accuracy and computational cost.

\section{References}

1. K. Carlberg, M. Khalil, K. Sargsyan, and S. Guzzetti. Uncertainty propagation in large-scale networks via Domain Decomposition. In preparation, 2017.

2. L. Mansilla Alvarez, P. Blanco, C. Bulant, E. Dari, A. Veneziani, and R. Feijóo. Transversally enriched pipe element method (TEPEM): An effective numerical approach for blood flow modeling. IJNMBE, 2017.

3. S. Guzzetti, S. Perotto, A. Veneziani. Hierarchical Model Reduction for Incompressible Fluids in Pipes. IJNME, 2017.

4. D. Xiu, S.J. Sherwin. Parametric uncertainty analysis of pulse wave propagation in a model of a human arterial network. JCP, 2007.

\section{Acknowledgements}

CNPq, FAPERJ, NSF Grant DMS-1419060.

Sandia National Laboratories is a multimission laboratory managed and operated by National Technology and Engineering Solutions of Sandia, LLC., a wholly owned subsidiary of Honeywell International, Inc., for the U.S. Department of Energy's National Nuclear Security Administration under contract DE-NA-0003525. 